\title{
Implementation of IoT Smart Blackout Curtain Including Voice Recognition Function
}

\author{
Minseong Kim, Yeji Kim, Yejin Yoon, and Hyenki Kim** \\ Andong National University, Korea \\ *hkkim@anu.ac.kr
}

\begin{abstract}
Smart blackout curtain automatically opens and closes according to the user's setting for the busy modern man's smooth sleep activity without needs to operate the curtain manually. In this paper, to open and close the curtain according to the temperature, illumination, voice recognition and fine dust, the blackout curtain utilizing IoT technology was implemented by adding the voice recognition technology to the opening function by each sensor, through which it is expected to provide the convenience and the benefits to the life by allowing the operation anywhere.
\end{abstract}

Keywords: Smart, IoT, Curtain, Voice, Recognition

\section{Introduction}

While the 4th industrial revolution is emerging, the products grafted with next-generation technologies are already being developed in many areas [1][2]. Among them, IoT refers to the technology or environment that transmits the data through internet in real-time by attaching the sensor to the object [3][4]. Since the daily routine of the modern man exposed to the excessive lights by the urbanization and the increase of night activities works as a cause of insufficient sleep, this study was intended to benefit the life by enhancing the quality of modern men's sleep using various sensors attachable to the Arduino so that they can sleep comfortably at least during the sleeping hours.

Measuring the fine dust through the fine dust immediately at the window sensor attached to the curtain and operating the air purifier connected to opening of curtain responding to the fine dust problem in our country and allowing the people to live in the healthy environment. Therefore, this paper was intended to provide the comfort to the life and allow the people to enjoy the healthy life by grafting the existing curtain with IoT, one of the next-generation technology, and the voice recognition function.

\section{Related Research}

In this chapter, the research was made to open and close the IoT blackout curtain using temperature and illumination sensor and to add the voice recognition function preparing the foundation for the advanced design. For the function of IoT blackout curtain, basically it is enabled to open and close automatically enhancing the user's convenience. And using the temperature and illumination sensors, opening and closing are possible according to the user's

Article history:

Received (November 7, 2019), Review Result (December 15, 2019), Accepted (January 28, 2020) 
set value. The user's convenience is enhanced further by adding the voice recognition function. The most distinguishing characteristic is that it can be managed in real-time by controlling it remotely through the application. In addition, it can be opened and closed more conveniently by the voice recognition function. The curtain can be opened and closed by measuring with the fine dust sensor and when the fine dust is higher than the set value, it will operate the air purifier.

\subsection{Voice Recognition API}

Application Program Interface (API) that supports the developer environment of the voice recognition program is a kind of collection of the functions required to develop the voice synthesis and voice recognition program [5]. Among the voice recognition technology, there is a technology to output the voice to texts with TTS technology but this study used mainly the speech recognizer API. Most of today's smartphones are equipped with voice recognition function. Apple is equipped with Siri, Samsung is equipped with Bigsby, Google is with Google Assistant, and there are Naver Clova, Giga Genie of KT, etc.

\subsection{IoT and Sensor}

The most important factor in selecting the sensor is the function, price and the size. The higher the performance, the lower the price and the smaller the size, in the wider area, the sensor is utilized and can generate more data. As an example of IoT sensor, there is the sensor for fine dust, light intensity at night, floating population, etc. and in this study, the sensors for illumination, temperature and fine dust were used. [Table 1] is the Main sensor used by industry [6].

Table 1. Main sensor used by industry

\begin{tabular}{|c|c|}
\hline \multirow{7}{*}{$\begin{array}{l}\text { Consumer- } \\
\text { electronics }\end{array}$} & Temperature sensor, Humidity sensor \\
\hline & Vibration sensor, Noise sensor \\
\hline & Contact sensor, touch sensor, Pressure sensor \\
\hline & Microphone, camera \\
\hline & Close proximity sensor, Illumination sensor, Color sensor \\
\hline & Acceleration, terrestrial magnetism, Gyroscope \\
\hline & IR movement, Body temperature sensor \\
\hline \multirow{5}{*}{ Home/ building } & Lane sensor \\
\hline & Carbon dioxide, fine dust, yellow dust detection \\
\hline & Gas, smoke, fire detection \\
\hline & Camera \\
\hline & Infrared lines, acoustic, vibration detection \\
\hline \multirow{5}{*}{ City/ environment } & Movement, pressure sensor \\
\hline & Barometric pressure, temperature, humidity \\
\hline & Sunshine, infrared lines, UV rays \\
\hline & Radiation detection sensor \\
\hline & Pesticide, toxic detection \\
\hline
\end{tabular}




\section{Demand Analysis}

The characteristics, the strength and weakness of the product and the application supported them related to the IoT business currently appeared in our country were analyzed and the characteristics differentiated of the smart blackout curtain being to be designed by benchmarking them.

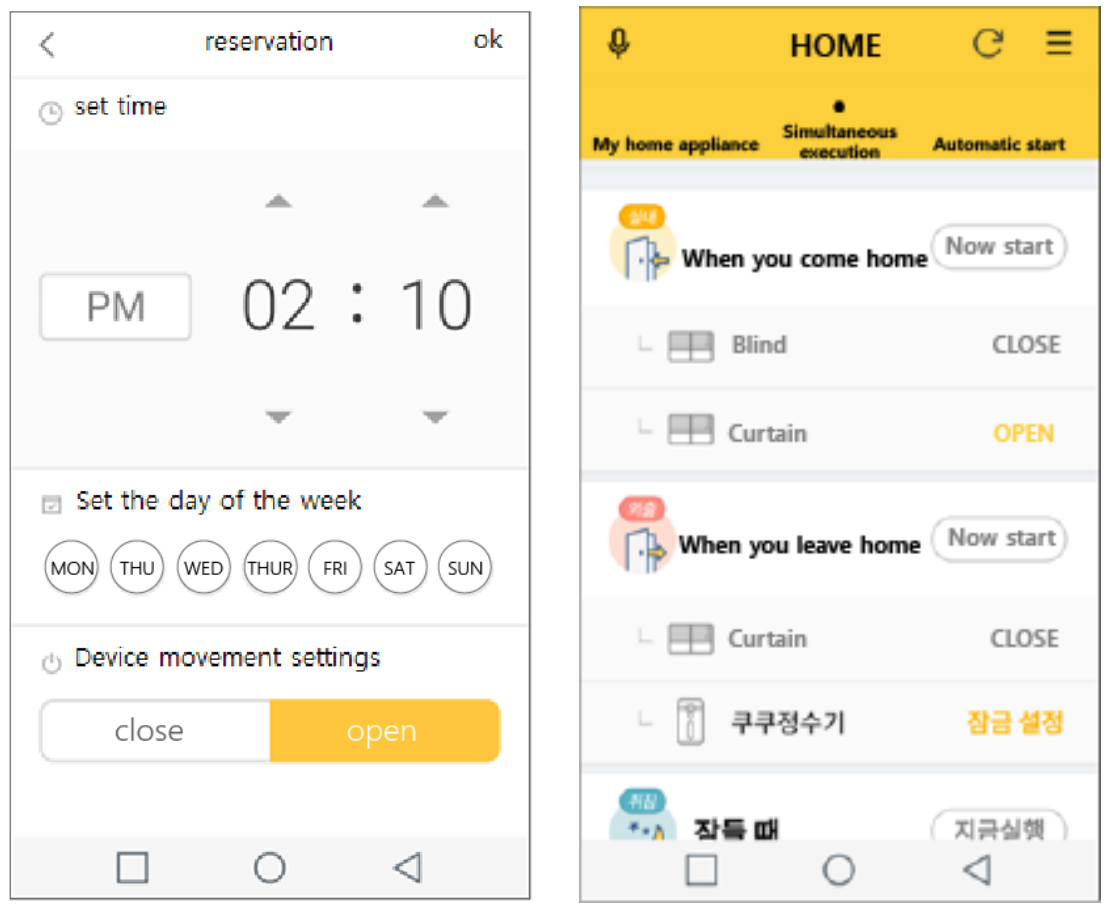

Figure 1. Application screen supporting IoT blinds of somfy and LGU+
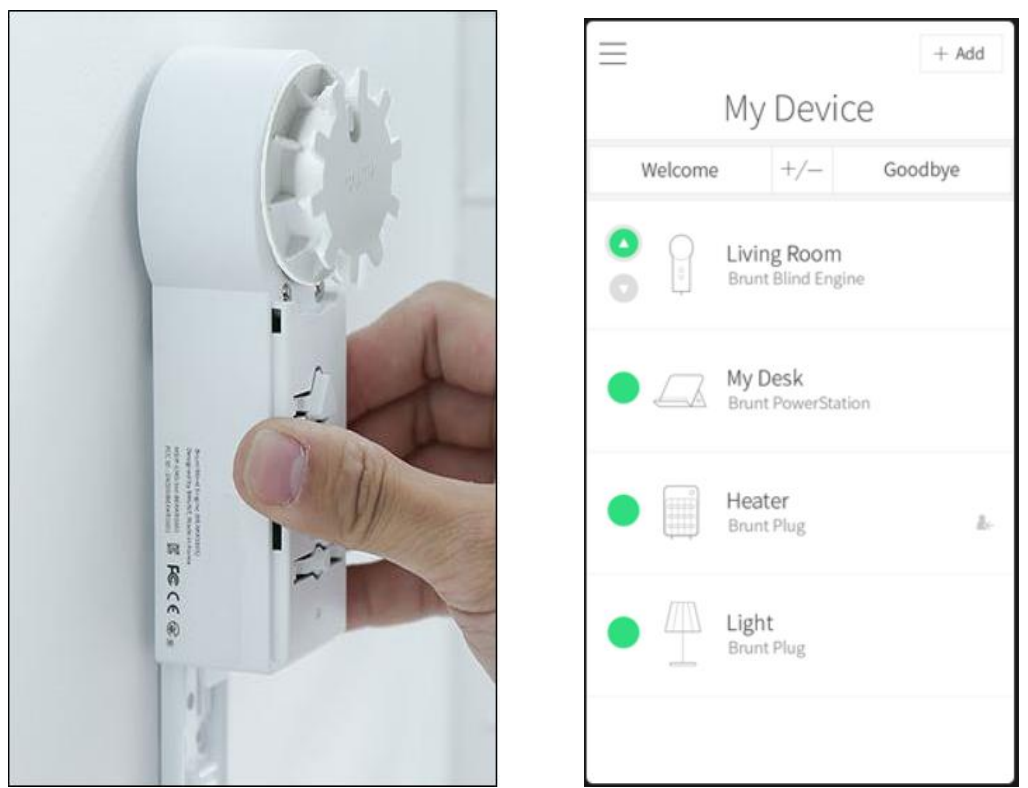

Figure 2. Brunt blind engine and support application screen 
[Figure 1] is the application supporting the blind of Somfy and LGU+ and can open and close the blind using smartphone anytime and anywhere [7]. Like the alarm function of the smartphone, it can open and close by setting the time. However, opening and closing operations are possible only through the smartphone application. In addition, it has weakness that it is the service required for the subscription of paid IoT service.

[Figure 2] is the Brunt Blind Engine and the supporting application. IoT blind of Somfy shown in Figure 1 requires the assistance of professional when installing it, but in case of Brunt Blind Engine, the engine can be attached easily alone without the assistance of professional, and it has advantage that the adjustment by the operation of slide as much as the user wants not by specific stage. However, it has disadvantage that the curtain made of cloth cannot be used and it is operated only with Amazon Echo.

In the results of analyzing these two products, the products related curtain $\&$ blind in the market can be opened and closed anytime and anywhere and the automatic operation enable to set alarm is basic. In addition, the neat and simple UI (User Interface) design contribute to using the application easily. However, the application has limitation such as operation and adjustment limited to the application, additional cost of paid service subscription, limited technical support and application. Therefore, we would like to design the smart blackout curtain that can be opened and closed automatically by product itself, without paying additional charge and supported without limitation. In addition, considering the seriousness of fine dust in our country, we would like to make it differentiated by adding the fine dust sensor.

\section{Design}

In this study, the main objective of the design is to design the smart blackout curtain that the curtain is operated automatically according to the setting or sensor operation not by the manual operation.

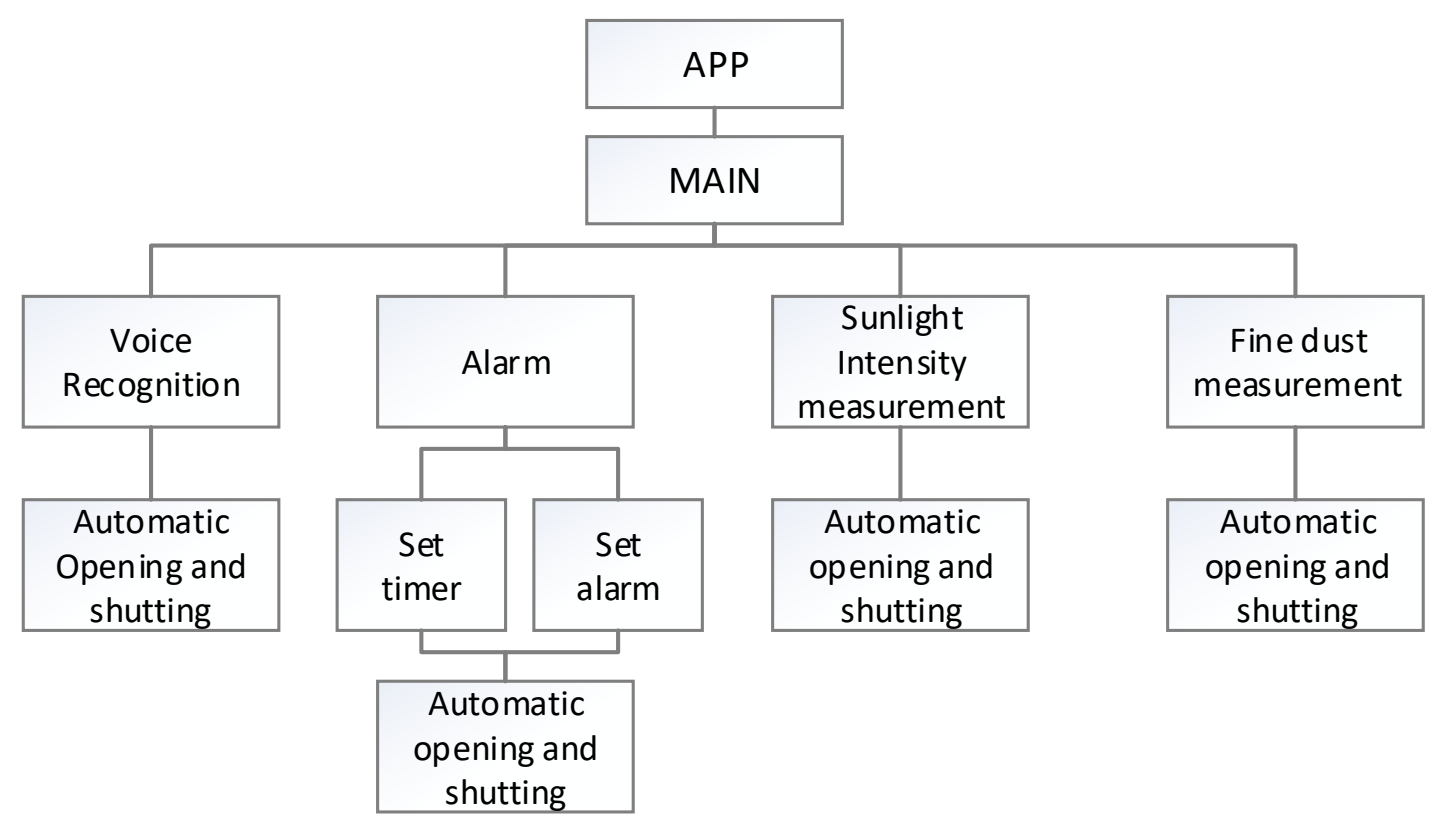

Figure 3. Flow chart 
As shown in [Figure 3], the temperature sensor, illumination sensor and the fine dust sensor measure the temperature, brightness and the fine dust concentration by detecting the surrounding environment. And then, it compares the information received from the sensors with the user setting in the application and opens or closes the curtain automatically. Opening and closing the curtain are possible through the voice of user by adding the voice recognition function although it does not reach the set value. [Figure 4] shows the artificial intelligence out of UI design of application. The relevant page can be viewed by setting in the main. The conversation in each situation of going out, sleep, the weather is added as a command. If the user speaks out command, the application execute the set command.

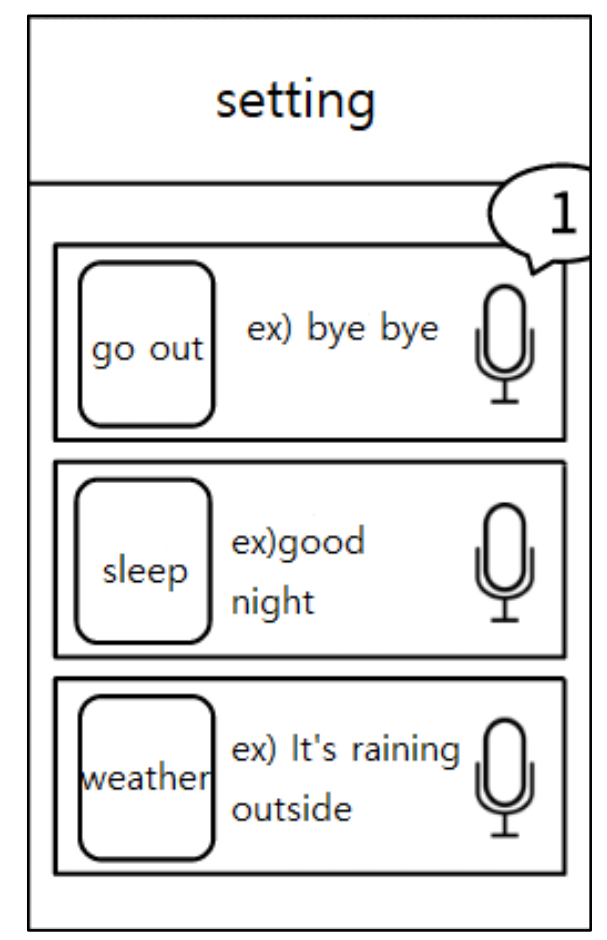

Figure 4. Voice recognition UI

\section{Conclusion}

In this paper, the smart blackout curtain, which opens and closes automatically by recognizing the light intensity, differentiated with existing blackout curtain, was designed. This blackout curtain can be controlled remotely through the application and equipped with voice recognition technology, the next-generation technology, to complement the inconvenience and something unsatisfactory. It was designed so that it can alleviate the inconvenience of opening and closing manually through the voice recognition, which is the core function, and can provide the convenience to the consumer with the function of opening and closing the curtain automatically by analyzing the intensity of light.

As the average temperature of the global village is raised continuously, the intensity of the sunlight becomes more stronger [8][9]. To get away the inconvenience such as waking up unintentionally by the sunlight or unable to see the object well by being dazzled, etc. the people who are using the blackout curtain are being increased. Therefore, this paper expect that the blackout curtain would be useful and convenient in our daily living by complementing and improving the existing blackout curtain. In addition, this paper intended 
to contribute to the growth of voice recognition technology increasing the interest and convenience of the consumer by equipping the voice recognition, which is an innovative technology, to general blackout curtain additionally.

\section{Acknowledgements}

This work was supported by a Research Grant of Andong National University.

\section{References}

[1] Jinhee Park, "Designing and implementing Android voice recording applications based on user calendar," Database research, vol.31, no.3, pp.147-158, (2015)

[2] Seungkwon Choi, "MultI-language automatic translation technique," Electronics and telecommunications trends, vol.20, no.5, October, pp.16-27, (2005)

[3] Y.T. Jeong, "The Sensor Physics Introduction,” Hongreung Science Publishers, Seoul, (2013)

[4] J. Shim, J. Ko, Y. Lee, and W. Jeong, "Funny Arduino," Seoul, Hanteemdeia, (2013)

[5] Su Hyeon Nam and Kyoo-Sung Noh, "A study on the effective approaches to big data planning," The Society of Digital Policy \& Management, vol.13, no.1, pp.227-235, (2015) DOI: 10.14400/JDC.2015.13.1.227

[6] Hojoong Lee, Hyunjung La, Changsup Keum, and Soodong Kim, "A process to design and implement service-based android applications," information processing society journal D, vol.18, no.4, pp.245-260, (2011) DOI: 10.3745/KIPSTD.2011.18D.4.245

[7] Shruti M. Patel and Shailaja Y. Kanawade, "Internet of things based smart home with intel Edison," the Advances in Intelligent Systems and Computing book series, pp.385-392, (2017)

[8] Y. Ha, Y. Kim, “A study on Interactive Costume Design based on Wearable Computer Technology," Journal of Korean Society Fashion Design, vol.14, no.1, pp.1-15, (2014)

[9] Minseong Kim, Yeji Kim, Yejin Yoon, and Hyenki Kim, "Design of IoT smart blackout curtain including voice recognition function $3^{\text {rd }}$," domestic and international integration conference, pp.19, (2019) 